\title{
Cycle Biologique en Conditions Semi-Naturelles de Plutella xylostella L., 1758 (Lepidoptères: Plutellidae), Insecte Ravageur du Chou Pomme en Côte d'Ivoire
}

\author{
Kouassi Adjoua Madeleine, \\ Université Peleforo Gon Coulibaly, Laboratoire de Biologie Végétale, \\ Production Végétale et Protection des Végétaux, IGA, Côte d'Ivoire

\section{Ouali-N'goran San-Whouly Mauricette,} \\ Université Félix Houphouet-Boigny, Laboratoire de Zoologie et Biologie \\ Animale,UFR Biosciences, Côte d'Ivoire \\ Soro Yadé René, \\ Université Félix Houphouet-Boigny, Laboratoire de Biotechnologie \\ UFR Biosciences, Côte d'Ivoire \\ Akessé Ettien Narcice, \\ Université Félix Houphouet-Boigny, Laboratoire de Zoologie et Biologie \\ Animale, UFR Biosciences, Côte d'Ivoire

\section{Coulibaly Adama,} \\ Université Félix Houphouet-Boigny, Laboratoire de Pharmacodynamie- \\ Biochimie UFR Biosciences, Côte d'Ivoire
}

Doi:10.19044/esj.2019.v15n27p326 URL:http://dx.doi.org/10.19044/esj.2019.v15n27p326

Résumé

Le chou pomme est la principale culture maraîchère pratiquée au nord de la Côte d'Ivoire. Plutella xylostella, son principal insecte ravageur, est présent sur la culture tout au long du cycle. Mais, les caractéristiques du cycle de développement de l'insecte restent peu connues en Côte d'Ivoire. L'objectif de cette étude était de déterminer le cycle de développement du ravageur à températures ambiantes. Elle a consisté en la caractérisation des stades de développement et en la détermination du taux d'émergence, du sex-ratio, de la fécondité et de la longévité des adultes. L'étude s'est déroulée de novembre 2017 à janvier 2018. Les insectes ont été élevés dans une cage cubique de 50 $\mathrm{cm}$ de côté dont les cadres sont en bois et les faces en grillage métallique. Trois générations du ravageur ont été observées. Les résultats ont montré que les quatre stades larvaires ont eu une durée moyenne de 3 à 4 jours pour les stades 1 et 2 , et 2 à 3 jours pour les stades 3 et 4 . La durée des stades larvaires a augmenté avec l'élevation de la température. La longévité des adultes a été de $11,98 \pm 0,64$ jours pour le mâle et 13,81 $\pm 0,45$ jours pour la femelle. Le taux 
d'émergence a été de $41 \%$. Le sex-ratio a été de 0,53 en faveur des femelles. La fécondité par femelle a été de 62,43 $\pm 15,62$ œufs. Les paramètres biologiques de Plutella xylostella ainsi obtenus peuvent orienter l'élaboration de stratégie de gestion de ce ravageur.

Mots-clés: Chou pomme, Plutella xylostella, Insecte ravageur, Cycle de développement

\title{
Biological Cycle in Semi-Natural Conditions of Plutella xylostella L., 1758 (Lepidoptera: Plutellidae), Insect Pest of Apple Cabbage in Côte d'Ivoire
}

Kouassi Adjoua Madeleine,

Université Peleforo Gon Coulibaly, Laboratoire de Biologie Végétale, Production Végétale et Protection des Végétaux, IGA, Côte d'Ivoire

\section{Ouali-N'goran San-Whouly Mauricette,}

Université Félix Houphouet-Boigny, Laboratoire de Zoologie et Biologie

Animale,UFR Biosciences, Côte d'Ivoire

\section{Soro Yadé René,}

Université Félix Houphouet-Boigny, Laboratoire de Biotechnologie

UFR Biosciences, Côte d'Ivoire

\section{Akessé Ettien Narcice,}

Université Félix Houphouet-Boigny, Laboratoire de Zoologie et Biologie

Animale, UFR Biosciences, Côte d'Ivoire

\section{Coulibaly Adama,}

Université Félix Houphouet-Boigny, Laboratoire de Pharmacodynamie-

Biochimie UFR Biosciences, Côte d'Ivoire

\begin{abstract}
Apple cabbage is the main vegetable crop grown in northern Côte d'Ivoire. Plutella xylostella, its main insect pest, is present on the crop throughout the cycle. But, the characteristics of the insect's development cycle remain poorly known in Côte d'Ivoire. This study aims to determine the development cycle of the pest at ambient temperatures. It consisted of characterizing developmental stages and determining the emergence rate, sex ratio, fecundity and longevity of adults. The study ran from November 2017
\end{abstract}


to January 2018. The insects were raised in a cubic cage of $50 \mathrm{~cm}$ sides with frames in wood and metal mesh faces. Three generations of the pest have been observed. The results showed that the four larval stages had an average duration of 3 - 4 days for stages 1 and 2, and 2 - 3 days for stages 3 and 4 . The duration of the larval stages has increased with the rise of the temperature. The longetivy of adults was $11.98 \pm 0.64$ days for the male and $13.81 \pm 0.45$ for the femelle. The emergence rate of adults was $41 \%$. The sex ratio was 0.53 in favor of females. Mean fertility per female was $62.43 \pm 15.62$ eggs. The biological parameters of Plutella xylostella obtained can guide the adoption of management strategy for this pest.

Keywords: Cabbage, Plutella xylostella, Insect pest, Development cycle

\section{Introduction}

Depuis les années 1990, la production de légumes frais s'est développée dans la région nord de la Côte d'Ivoire (Le Guen, 2004). Cette activité maraîchère revêt une importance capitale car elle participe à la sécurité alimentaire et à la réduction de la pauvreté (Fondio et al., 2007 ; Mahyao et $a l ., 2009)$. Parmi ces cultures maraichères, le chou occupe la première place (Tuo et al., 2017). La culture du chou fait malheureusement l'objet d'une forte pression parasitaire essentiellement due aux insectes ravageurs. Parmi ces insectes, la teigne du chou Plutella xylostella constitue le principal ravageur (Kouakou et al., 2002 ; Glitho et al., 2008 ; Douan et al., 2013). Ce ravageur prolifère dans toutes les cultures de chou grace à son cycle de développement très court, sa capacité à s'adapter à une large gamme de température, sa fécondité élevée et sa résistance à plusieurs familles chimiques d'insecticide (Furlong et al., 2013). L'élaboration de stratégies de luttes efficaces et durables contre ce ravageur requiert la connaissance de sa bio-écologie.

Des études ont été réalisées sur la biologie de $P$. xylostella en laboratoire (Ngowi et al., 2017 ; Saeed et al., 2018). Elles ont prouvé que la durée du cycle de développement de ce ravageur variait avec la température et la source d'alimentation. En milieu naturel, la température varie. Cette fluctuation peut modifier la durée du cycle. L'insuffisance de données sur la biologie de P. xylostella en Côte d'Ivoire justifie le choix de cette étude. L'objectif est de déterminer quelques paramètres biologiques de $P$. xylostella en conditions semi-ambiantes. Spécifiquement, il s'est agi de déterminer la durée des stades de développement, le taux d'émergence et la longévité des adultes, le sex-ratio et la fécondité des femelles. 


\section{Matériel et Méthodes \\ Matériel d'élevage}

L'élevage a été réalisé dans une cage cubique de $50 \mathrm{~cm}$ de côté. Les cadres étaient en bois et les faces en grillage métallique de $0,4 \mathrm{~mm}$. L'une des faces latérales a été munie d'une ouverture carrée de $30 \mathrm{~cm}$ de côté permettant les manipulations à l'intérieur de la cage. Les pieds de la cage sont plongés dans des boîtes contenant de l'eau savonneuse pour éviter les attaques des fourmis. Un thermo-hygromètre a permis de relever la température et l'humidité relative dans la cage.

\section{Conduite de l'élevage de $P$. xylostella}

L'étude s'est déroulée à Korhogo, localité située au nord de la Côte d'Ivoire (entre $8^{\circ} 26$ et $10^{\circ} 27$ de latitude Nord et $5^{\circ} 17$ et $6^{\circ} 19$ de longitude Ouest). Elle a été menée de novembre 2017 à janvier 2018.

Les spécimens étaient des larves de $P$. xylostella collectées sur les plants de chou pomme du site expérimental de l'Université Peleforo Gon Coulibaly à Korhogo. Les larves ont été mises en élevage sur des feuilles de chou pomme jusqu'à l'obtention des adultes. Dix mâles et dix femelles de cette population ont constitué l'échantillon de départ de l'étude.

Les premières observations ont commencé avec l'émergence des adultes issus des spécimens. La cage a été posée en milieu ambiant. Les dix couples ont été introduits dans la cage contenant trois plants de chou de huit semaines. Ces plants ont servi de pondoirs. Les adultes ont été nourris avec de l'eau contenant $10 \%$ de miel (Jaleel et al., 2017 ; Saeed et al., 2018). Cette solution a été fournie dans du coton imbibé. A l'éclosion des œufs, les plants ont été sortis de la cage. Cinq feuilles contenant des larves ont été détachées des plants et introduites dans la cage (Figure 1). Chaque feuille contenait dix larves (Figure 2). Cinq nouvelles feuilles fraîches de chou ont été déposées dans la cage toutes les 24 heures pour l'alimentation des larves. L'expérience a été repetée sur trois générations. Pour chaque génération, 10 couples puis 50 larves sont suivis. La température moyenne a été de $25,6 \pm 7,3{ }^{\circ} \mathrm{C}$. Les températures extrêmes ont oscillé entre 15 et $37^{\circ} \mathrm{C}$. L' humidité relative a varié de 34 à $61 \%$ avec une moyenne de $41 \pm 8,4 \%$.

\section{Paramètres étudiés}

Les observations se sont déroulées de la ponte des œufs jusqu'à la mort des imagos. Les paramètres étudiés sont définis ci-dessous.

- Période de pré-oviposition

C'est le temps écoulé entre l'accouplement et la première ponte. Des observations préliminaires ont permis de constater que les femelles s'accouplaient et pondaient leurs premiers œufs à proximité de leur mue imaginale (Figure 3). La date et 1'heure de l'accouplement ainsi que celles de 
la première ponte ont été notées pour chaque femelle. Chaque cage contenait dix femelles.

- Période de ponte

Elle correspondait au temps écoulé entre la première et la dernière ponte.

- Durée d'incubation

Les supports de ponte ont été inspectés régulièrement. A chaque passage, la date, l'heure et le nombre d'oeufs pondus ont été notés jusqu'au jour où il n'y avait plus de nouvelles pontes. Le nombre d'éclosion a été également noté à chaque passage jusqu'à ce qu'il n'y ait plus d'émergence de larve de stade 1 . La durée moyenne d'incubation DI a été calculée selon la formule :

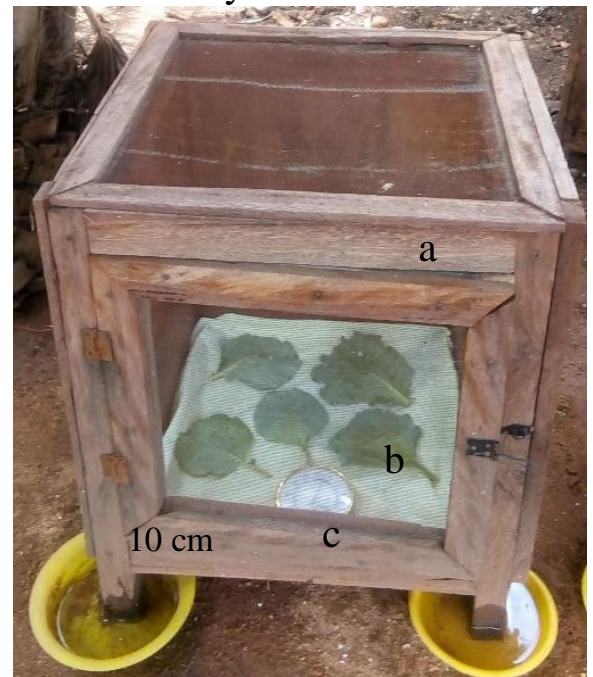

\section{Légende :}

a : cage d'élevage

$\mathrm{b}$ : feuille de chou

$c$ : thermo-hygromètre

Figure 1 : cage d'élevage contenant cinq feuilles de chou et un thermo-hygromètre

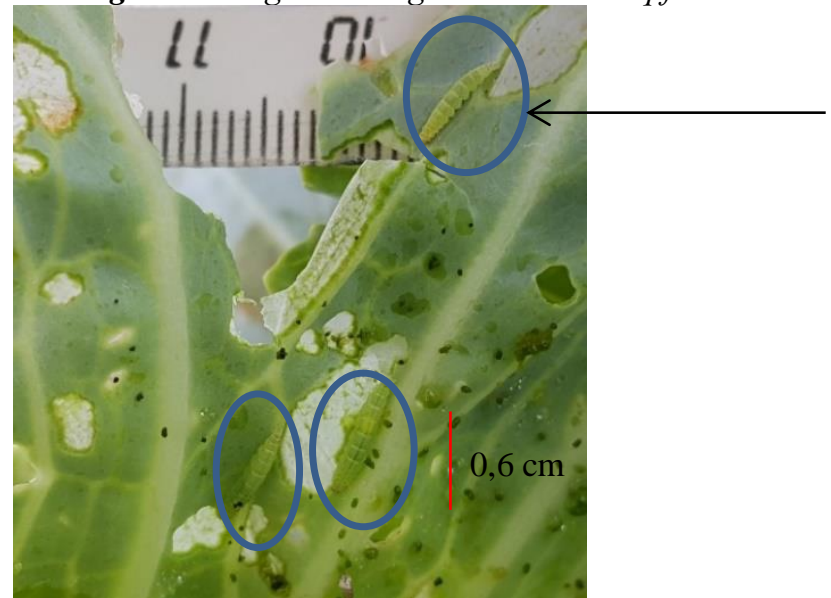

Larve de P. xylostella

Figure 2 : Larves de P. xylostella sur une feuille de chou pomme 


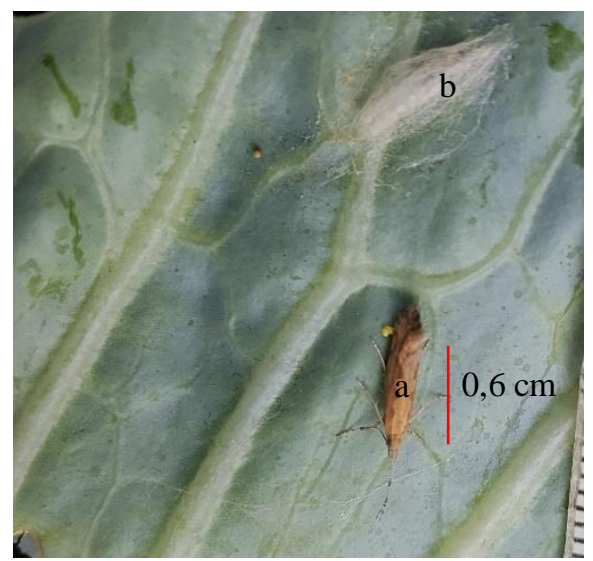

Figure 3 : femelle de P. xylostella en ponte (a) à proximité de son exuvie (b)

$$
\mathrm{DI}=\frac{\sum \mathrm{n}_{\mathrm{i}} \mathrm{e}^{*} \mathrm{~d}_{\mathrm{i}}}{\sum \mathrm{n}_{\mathrm{i}} \mathrm{e}} \quad \begin{array}{r}
\mathrm{n}_{\mathrm{i}} \mathrm{e}: \text { nombre d'éclosion à la date } \mathrm{i} \\
\mathrm{d}_{\mathrm{i}}: \text { durée d'éclosion à la date } \mathrm{i}
\end{array}
$$

- Durée des stades de développement

Les larves de stade 1 vivaient à l'interieur du parenchyme des feuilles. Leur durée de développement a été estimée au temps mis entre la date de l'éclosion et l'apparition des larves à la face inférieure des feuilles de chou. Le passage du stade 2 aux stades 3 et 4 est caractérisé par des mues. Le nombre de nouvelles mues est noté à chaque passage. Le temps entre deux mues successives est noté et la moyenne est calculée pour chaque stade. Le temps mis entre le dernier stade larvaire et l'émergence des adultes est également noté. La durée moyenne de chaque stade de développement (Dd) est calculée selon la formule ci-dessous :

$\mathrm{Dd}=\sum\left(\mathrm{X}_{\mathrm{i}} \times \mathrm{n}_{\mathrm{i}}\right) / \sum \mathrm{n}_{\mathrm{i}}$

$\mathrm{X}_{\mathrm{i}}=$ durée/stade/individu

$\mathrm{n}_{\mathrm{i}}=$ effectif d'individus

considérés pour chaque stade

- Taux d'émergence

Le nombre d'adultes émergés est relevé chaque jour jusqu'à ce qu'il n'y ait plus de sortie. Le taux d'émergence a traduit le pourcentage d'imagos émergés sur le nombre total d'œufs pondus.

- Sex-ratio

Le sex-ratio a exprimé la proportion des individus de sexe masculin ou féminin sur le nombre total d'individus.

- Temps de maturation sexuelle

La larve de sexe mâle est identifiable par la présence d'une tache blanchâtre sur le cinquième segment abdominal. La tache révèle la présence de gonades. Cette caractéristique permet de former dix couples au stade nymphal. La date et l'heure de l'émergence des imagos sont notées. Celles des accouplements 
sont également notées. Les femelles ne s'accouplent qu'une fois, contrairement aux mâles qui peuvent le faire deux à trois fois (Talekar \& Shelton, 1993). Le temps moyen de maturation sexuelle correspond au temps mis entre l'émergence des adultes et leur accouplement.

- Fécondité

La fécondité est considérée comme étant le nombre de pontes par une femelle au cours de sa vie. Il y a dix femelles par génération. Le nombre de pontes est noté chaque jour jusqu'à ce qu'on n'en observe plus. La fécondité est déterminée par le nombre total d'œufs sur le nombre de femelles.

- Longévité des imagos

C'est le temps de vie de l'insecte de la mue imaginale à sa mort. Le nombre d'imagos morts est relevé chaque jour jusqu'à la mort du dernier individu.

La durée moyenne de vie (DV) des imagos est déterminée à partir des résultats obtenus en faisant la somme des différentes durées de vie que l'on divise par le nombre total d'individus ayant atteint le stade imaginal.

DV $\frac{\sum \mathrm{X}_{\mathrm{i}} \mathrm{n}_{\mathrm{i}}}{\sum \mathrm{n}_{\mathrm{i}}}$

- Taux de fertilité

C'est le pourcentage d'œufs éclos par rapport au nombre total d'œufs pondus.

- Durée du cycle de développement

C'est le temps écoulé entre la date de ponte et l'obtention du stade adulte.

\section{Analyse des données}

Pour les différents paramètres observés, les données des trois générations ont été comparées à l'aide du test non paramétrique de Kruskalwallis au seuil de $5 \%$. Le test de corrélation de Spearman $(R)$ a été utilisé pour déterminer l'influence de la température sur la durée des stades de développement. Lorsque la tendance entre les deux variables n'était pas monotone, $R$ a pris une valeur proche de 0 . La tendance est croissante lorsque $0<R \leq 1$; elle est décroissante lorsque $0>R \geq-1$. Toutes ces analyses ont été effectuées à l'aide du logiciel Statistica version 7.1.

Résultats

P. xylostella a un cycle de développement qui a duré de 15,39 $\pm 1,54$ jours à 19,42 $\pm 0,16$ jours (Figure 4 ) et un stade imaginal de 12,74 $\pm 0,64$ jours. La durée du cycle de vie a varié de $28,13 \pm 0,12$ jours à 32,16 $\pm 0,27$ jours.

\section{Qufs}

Les œufs étaient jaunes, ovales et allongés. Ils ont mesuré $0,5 \pm 0,01$ $\mathrm{mm}$. Ces œufs ont été pondus par petits groupes de 7 à 10 à la face inférieure 
des feuilles. Le taux de fertilité a été de $87 \%$. Le temps d'incubation a été de 2,71 $\pm 0,18$ jours (Tableau 1 ).

Tableau 1 : Durée moyenne d'incubation des oufs de P. Xylostella

\begin{tabular}{|c|c|c|c|c|}
\hline \multirow[t]{2}{*}{ Génération } & \multicolumn{2}{|c|}{ Température $\left({ }^{\circ} \mathrm{C}\right)$} & \multirow{2}{*}{$\begin{array}{l}\text { Nombre d'oufs } \\
\text { par femelle }\end{array}$} & \multirow{2}{*}{$\begin{array}{l}\text { Durée d'incubation } \\
\text { (jours) }\end{array}$} \\
\hline & Min. & Max. & & \\
\hline 1 & 16 & 33 & $57 \pm 0,62$ & $2,75 \pm 0,20$ \\
\hline 2 & 15 & 34 & $68 \pm 0,37$ & $2,74 \pm 0,12$ \\
\hline 3 & 15 & 37 & $62 \pm 0,47$ & $2,64 \pm 0,20$ \\
\hline Moyenne & & & $62,43 \pm 15,62$ & $2,71 \pm 0,180$ \\
\hline
\end{tabular}

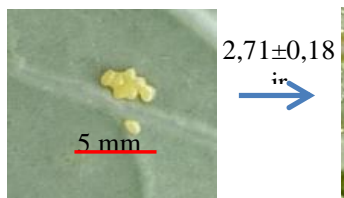

Eufs
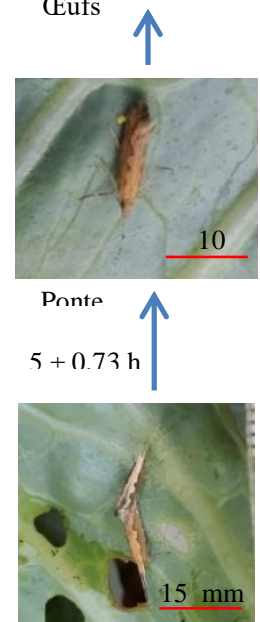

Accouplemen
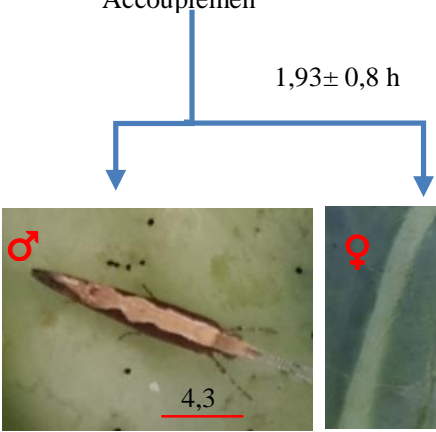

Adulte

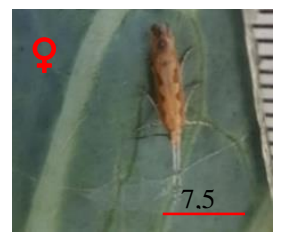

Adult

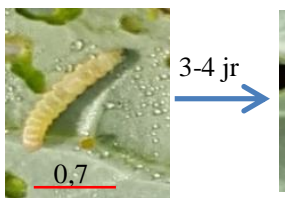

I arve de stade 1

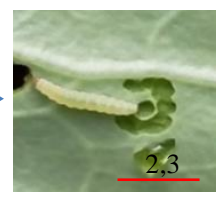

Larve de stade 2

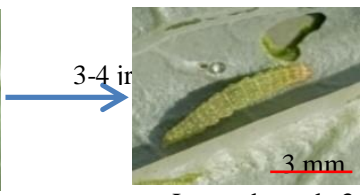

Larve de stade 3 $2-3$ ir

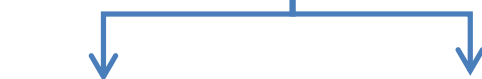

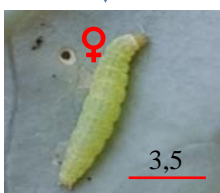

Larve de stade 4
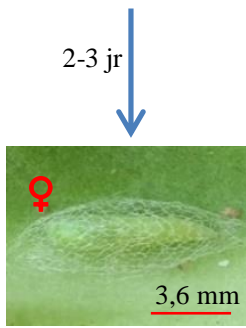

Pré-nymphe
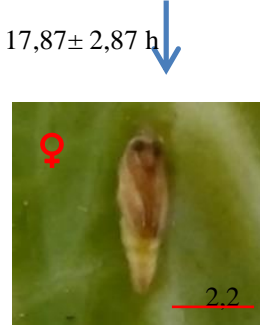

Nvmnhe

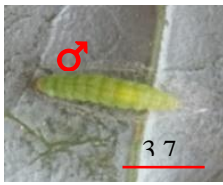

Larve de stade 4

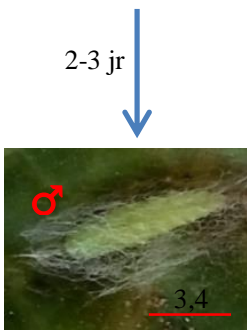

Pré-nymphe $17,87 \pm 2,87 \mathrm{~h}$

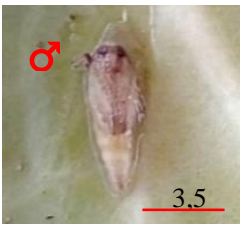

Nvmphe

Figure 4 : Cycle de devéloppement de P.xylostella (Lepidoptères : Plutellidae).

Température moyenne : $25,64 \pm 7,3{ }^{\circ} \mathrm{C}$; Humidité relative : $41 \pm 8,4 \%$.

$$
j r=\text { jour } ; h=\text { heure }
$$




\section{Caractéristiques des stades larvaires}

Quatre stades larvaires ont été déterminés (Tableau 2). A l'éclosion, les larves de premier stade étaient translucides. Elles ont mesuré en moyenne $1,2 \pm 0,06 \mathrm{~mm}$. Les larves de deuxième stade étaient de couleur jaune pâle. Elles ont eu une taille moyenne de 3,5 $\pm 0,90 \mathrm{~mm}$. Au troisième stade larvaire, les larves ont eu une coloration vert foncée avec une forte pilosité. Elles ont mesuré $6 \pm 0,41 \mathrm{~mm}$. Au dernier stade larvaire, la taille des larves a été de $8 \pm$ $0,96 \mathrm{~mm}$. Les stades larvaires 1 et 2 ont duré 3 à 4 jours. Les stades larvaires 3 et 4 ont eu une durée de 2 à 3 jours (Tableau 2). Il existe une forte corrélation positive (Tableau 3 ) entre les durées des stades larvaires et les variations de température.

Tableau 2 : Durée moyenne des stades larvaires de P. xylostella

\begin{tabular}{cccccc}
\hline \multicolumn{2}{c}{ Température $\left({ }^{\circ} \mathbf{C}\right)$} & \multicolumn{5}{c}{ Durée des stades larvaires (jours) } \\
\hline Min. & Max. & Stade 1 & Stade 2 & Stade 3 & Stade 4 \\
\hline 16 & 33 & $3,46 \pm 0,08$ & $3,2 \pm 0,1$ & $2,30 \pm 0,08$ & $2,43 \pm 0,10$ \\
15 & 34 & $3,60 \pm 0,11$ & $3,37 \pm 0,10$ & $2,85 \pm 0,04$ & $2,55 \pm 0,10$ \\
15 & 37 & $4,02 \pm 0,22$ & $3,76 \pm 0,11$ & $3,22 \pm 0,38$ & $2,90 \pm 0,28$ \\
\hline
\end{tabular}

Tableau 3 : Corrélation entre la température et la durée des stades larvaires

\begin{tabular}{ccccc}
\hline Corrélation & Stade 1 & Stade 2 & Stade 3 & Stade 4 \\
\hline $\mathrm{p}$ & 0,00 & 0,00 & 0,00 & 0,00 \\
$R$ & 0,96 & 0,94 & 0,83 & 0,97 \\
\hline
\end{tabular}

\section{Nymphose}

A la fin du quatrième stade du développement larvaire, la larve a arrêté de se nourrir et a tissé autour d'elle, un cocon de soie lâche et blanchâtre. Cette activité s'est effectuée en $0,74 \pm 0,12$ jours (Tableau 4). C'est la prénymphose. La coloration verte de la larve est passée progressivement au brun au stade nymphal. La nymphe a mesuré en moyenne $7 \pm 0,02 \mathrm{~mm}$. Au bout de $1,94 \pm 0,11$ jours, l'adulte qui est un papillon, a émergé du cocon.

Tableau 4 : Durée moyenne des stades pré-nymphal et nymphal de P. xylostella

\begin{tabular}{llcll}
\hline Génération & \multicolumn{2}{l}{ Température $\left({ }^{\circ} \mathbf{C}\right)$} & \multicolumn{2}{l}{ Durée de développement (jours) } \\
\cline { 2 - 5 } & Min. & Max. & Pré-nymphe & Nymphe \\
\hline 1 & 16 & 33 & $0,74 \pm 0,12$ & $1,94 \pm 0,08$ \\
2 & 15 & 34 & $0,74 \pm 0,10$ & $1,94 \pm 0,10$ \\
3 & 15 & 37 & $0,74 \pm 0,13$ & $1,94 \pm 0,15$ \\
\hline Moyenne & & & $0,74 \pm 0,12$ & $1,94 \pm 0,11$ \\
\hline
\end{tabular}

\section{Stade imaginal}

L'adulte de $P$. xylostella a mesuré $15 \pm 0,09 \mathrm{~mm}$ d'envergure. Il est brun grisâtre, caractérisé par des motifs en losange. Ces motifs étaient de couleur crème chez le mâle et jaune chez la femelle. Ils étaient apparus sur les ailes repliées. Le taux d'émergence a été de $41 \%$. Le sex-ratio a été de 0,53 en faveur des femelles. L'accouplement a eu lieu environ deux heures après 
l'émergence. La femelle a commencé à pondre le jour même. Les pontes se sont étendues sur $8 \pm 0,41$ jours. La fécondité des femelles a été de $62,43 \pm$ 15,62 œufs. La longévité a varié selon le sexe $(\mathrm{H}=225,70 ; \mathrm{p}=0,00)$. Les mâles ont vécu en moyenne 11,98 $\pm 0,64$ jours tandis que les femelles ont vécu 13,81 $\pm 0,45$ jours (Tableau 4).

Tableau 5 : Longévité moyenne des adultes de P. Xylostella

\begin{tabular}{llllll}
\hline Génération & \multicolumn{2}{l}{ Température $\left({ }^{\circ} \mathbf{C}\right)$} & & \multicolumn{3}{l}{ Longévité par sexe (jours) } \\
\cline { 2 - 3 } \cline { 5 - 6 } & Min. & Max. & & Mâle & Femelle \\
\hline 1 & 16 & 33 & & $12,00 \pm 0,63$ & $13,83 \pm 0,27$ \\
2 & 15 & 34 & & $11,98 \pm 0,62$ & $13,80 \pm 0,57$ \\
3 & 15 & 37 & & $11,95 \pm 0,68$ & $13,81 \pm 0,47$ \\
\hline Moyenne & & & $11,98 \pm 0,640$ & $13,81 \pm 0,450$ \\
\hline
\end{tabular}

\section{Discussion}

L'élevage de $P$. xylostella à $25,6 \pm 7,3{ }^{\circ} \mathrm{C}$ a permis d'identifier quatre stades larvaires d'une durée moyenne de 10 à 14 jours. Ces valeurs sont plus élevées que celles obtenues à $25 \pm 1{ }^{\circ} \mathrm{C}$ par Alizadeh et al. (2011), Ngowi et al. (2017) et Saeed et al. (2018). Ces auteurs ont obtenu respectivement 8,88 jours, 7,89 jours et 7,82 jours. Les écarts observés pourraient provenir de la variation de la température observée dans la présente étude (écart type $=7,3$ $\left.{ }^{\circ} \mathrm{C}\right)$. Les différences pourraient également s'expliquer par la corrélation positive qui a existé entre la température et la durée des stades larvaires alors que Ngowi et al. (2017) et Saeed et al. (2018) avaient montré que l'augmentation de la température entrainait la reduction de la durée des stades larvaires.

La longévité des adultes a été plus courte chez le mâle $(11,98 \pm 0,64$ jours) que chez la femelle (13,81 $\pm 0,68$ jours). Gowri et Manimegalai (2016) ont également montré que la femelle vivait plus longtemps (16 jours) que le mâle (12 jours). Cependant, nos résultats diffèrent de ceux de plusieurs auteurs (Soufbaf et al., 2010 ; Alizadeh et al., 2011 ; Fathi et al., 2011 ; Ngowi et al., 2017 ; Saeed et al., 2018) qui ont mentionné des longévités plus élevées chez le mâle que chez la femelle. Cette différence pourrait s'expliquer par le sexratio qui a été plus élevé dans la présente étude en faveur des femelles $(0,53)$ alors qu'elle était de 0,48 selon Alizadeh et al. (2011). La différence pourrait également être due à la qualité de la nourriture. En effet, contrairement aux précedents travaux où les repas étaient synthétiques ou à base de chou-fleur, dans notre présente étude, les larves ont été nourries avec des feuilles fraîches de chou pomme.

La fécondité par femelle a été de $62,43 \pm 15,62$ œufs. Le nombre d'oeufs pondus a été plus faible que ceux obtenus à $25^{\circ} \mathrm{C}$ par Ngowi et al. (2017) et Saeed et al. (2018). Ces derniers ont montré que la fécondité par femelle était respectivement de $197,83 \pm 14,39$ et $261,93 \pm 2,68$ œufs. Cette faible fécondité pourrait être imputable aux températures maximales. Ces 
dernières ont atteint des valeurs supérieures à $35^{\circ} \mathrm{C}$, valeur à partir de laquelle la ponte est intérrompue (Ngowi et al., 2017). Un effet de masse au niveau des larves pourrait également être mis en cause. En effet, bien qu'elles puissent vivre à quelques millimètres les unes des autres, Castelo-Branco et Gatehouse (1999) ont montré que lorsque les larves atteignent une densité trop élevée, les femelles obtenues ont souvent des ovaires atrophiés. Elles pondent donc moins d'œufs.

\section{Conclusion}

Le développement des quatre stades larvaires de $P$. xylostella s'est déroulé sur 10 à 14 jours. La durée de chacun des stades larvaires a augmenté avec la température. Le cycle de vie du ravageur a eu une durée totale de 28,13 $\pm 0,12$ jours à $32,16 \pm 0,27$ jours. Il y a donc le risque qu'à une température moyenne de $25,6 \pm 7,3^{\circ} \mathrm{C}$, il y ait deux à trois générations par cycle cultural. La connaissance de la durée des stades larvaires aidera à prévoir la durée de nuisibilité du ravageur et à adopter une méthode de lutte appropriée.

\section{References :}

1. Alizadeh, M., Rassoulian, G. R., Karimzadeh, J., Hosseini-Naveh, V., \& Farazmand, H. (2011). Biological Study of Plutella xylostella (L.) (Lep: Plutellidae) and It's Solitary Endoparasitoid, Cotesia vestalis (haliday) (Hym. Braconidae) under Laboratory Conditions. Pakistan Journal of Biological Sciences. · DOI: 10.3923/pjbs.2011.1090.1099.

2. Castelo-Branco, M., Gatehouse, A. G. (1999). Food availability and larval density affect ovarian development in Plutella xylostella (L.) (Lepidoptera: Yponomeutidae). Anais da Sociedade Entomologica do Brasil. 28: 611-616.

3. Douan, B. G., Doumbia, M., Kouadio, D., Kra, V. M., Dagnogo, M. (2013). Comparaison de la dynamique des populations de Spodoptera littoralis (Boisduval) (Lepidoptera : Noctuidae) à celles de deux lépidoptères du chou dans le district d'Abidjan en Côte d'Ivoire. Journal of Animal and Plant Sciences. Vol.17, Issue 1: 2412-2424.

4. Fathi, S. A. A., Bozorg-Amirkalaee, M., et Sarfaraz, R. M. (2011). Preference and performance of Plutella xylostella (L.) (Lepidoptera: Plutellidae) on canola cultivars. Journal of Pests Sciences., 84: 41-47.

5. Fondio, L., Kouamé, C., N'Zi, J. C., Mayhyao, A., Agbo, E., et Djidji, A. H. (2007). Survey of indigenous leafy vegetable in the urban an periurban areas of Côte d'Ivoire. In: M.I. Chadha et al. (EDS). Indigenous vegetables and legumes: prospects for fighting poverty, hunger and malnutrition. Acta horticulturae. 752, pp 287-289.

6. Furlong, M. J., Wright, D. J., Dosdall, L. M. (2013). Diamondback moth ecology and management: problems, progress, and prospects. 
Annual Review of Entomology. 58: 517-541. https://doi.org/10.1146/ annurev-ento120811-153605 PMID: 23020617.

7. Glitho, I. A., Ketoh, K. G., Nuto, P. Y., Amevoin, S. K., et Huignard, I. (2008). Approches non toxiques et non polluantes pour le contrôle des populations d'insectes nuisibles en Afrique du centre et de l'ouest ; 207-217. In Biopesticides d'origine végétale ( $2^{\mathrm{e}}$ éd), Regnault-Roger C., Philogène B. J. R., Vincent C. (eds). Lavoisier, TEC \& DOC : Paris. 550p.

8. Gowri, G., \& Manimegalai, K. (2016). Biology of diamondback moth, Plutella xylostella (Lepidoptera: Plutellidae) of cauliflower under laboratory condition. Intern. Journal of Fauna and Biological Studies. 3(5): 29-31.

9. Jaleel, W., Saeed, S., Saeed, Q., Naqqash, M. N., Sial, M. U., Aine, Q. U. (2017). Effects of three different cultivars of cruciferous plant on the age-stage, two-sex life table traits of Plutella xylostella (L.)(Lepidoptera: Plutellidae). Entomological Research. Cited by Saeed, S., Jaleel, W., Naqqash, M. N., Saeed, Q., Zaka, S. M., Sarwar, Z. M., Ishtiaq, M., Qayyum, M. A., Sial, M. U., Aine Q. U., Batool, M., Khan, K. A., Ghramh, H. A., Hafeez, M., Ansari, M. J., Sharma, J. K. (2018). Fitness parameters of Plutella xylostella (L.) (Lepidoptera; Plutellidae) at four constant temperatures by using age-stage, two-sex life. Saudi Journal of Biological Sciences. https://doi.org/ 10.1016/j.sjbs. 2018.08.026.

10. Kouakou, A. E., Cissé G., Doumbia M., Girardin O. (2002). Contraintes parasitaires des cultures maraîchères en Côte d'Ivoire. Boiterre, Revue internationale Sciences de la Vie et de la Terre, $\mathrm{N}^{\circ}$ spécial. Actes du colloque international, Centre suisse du 27-29 août 2001.

11. Le Guen, T. (2004). Le développement agricole et pastoral du nord de la Côte d'Ivoire : problème de coexistence. Les cahiers d'Outre-mer 2 : $\mathrm{N}^{\circ} 226-227.200$ p. DOI : 10.4000/com.563.

12. Mahyao, A., Kouamé, C., Agbo, E., N'Zi, J.C., Fondio, L., et Van, D. P. (2009). Socio-economic importance of urban market supply chains and indigenous leafy vegetables of Côte d'Ivoire. Acta Horticulturae, 806: 489-496.

13. Ngowi, B. V., Tonnang, H. E. Z., Mwangi, E. M., Johansson, T., Ambale, J., Ndegwa, P.N., Subramanian, S. (2017). Temperaturedependent phenology of Plutella xylostella (Lepidoptera: Plutellidae): Simulation and visualization of current and future distributions along the Eastern Afromontane. PLoS ONE 12(3): e0173590. https://doi.org/10.1371/journal. pone.0173590. 
14. Saeed, S., Jaleel, W., Naqqash, M. N., Saeed, Q., Zaka, S. M., Sarwar, Z. M., Ishtiaq, M., Qayyum, M. A., Sial, M. U., Aine Q. U., Batool, M., Khan, K. A., Ghramh, H. A., Hafeez, M., Ansari, M. J., Sharma, J.K. (2018). Fitness parameters of Plutella xylostella (L.) (Lepidoptera; Plutellidae) at four constant temperatures by using agestage, two-sex life. Saudi Journal of Biological Sciences https://doi.org/ 10.1016/j.sjbs.2018.08. 026.

15. Soufbaf, M., Fathipour, Y., Karimzadeh, J., and Zalucki, M. P. (2010). Development and age-specific mortality of Diamondback moth on Brassica host plants. Pattern and causes of mortality under laboratory conditions. Annals of the Entomological Society of America., 103: 574-579.

16. Talekar, N. S, and Shelton, A. M. (1993). Biology, ecology, and management of the diamondback moth. Annual Review of Entomology. 38:275/301.

17. Tuo, Y., Dago, D. N., Yapo, L. M., and Koua, K. H. (2017). Screening of phytosanitary practices in vegetable growth activities northern of Côte D'Ivoire. International Journal of Recent Scientific Research. 8: 17396-17402. 